\title{
Sensor-enhanced 3D conformal cueing for safe and reliable HC operation in DVE in all flight phases
}

\author{
Thomas Münsterer*a, Tobias Schafhitzel ${ }^{\mathrm{a}}$, Michael Strobel ${ }^{\mathrm{a}}$, Philipp Völschow ${ }^{\mathrm{a}}$, \\ Stephanus Klasen ${ }^{\mathrm{a}}$, Ferdinand Eisenkeil ${ }^{\mathrm{b}}$ \\ aAirbus Defence and Space, Situational Awareness Solutions, 88039 Friedrichshafen, Germany; \\ ${ }^{b}$ University of Konstanz, Universitätsstraße 10, 78464 Konstanz, Germany
}

\begin{abstract}
Low level helicopter operations in Degraded Visual Environment (DVE) still are a major challenge and bear the risk of potentially fatal accidents. DVE generally encompasses all degradations to the visual perception of the pilot ranging from night conditions via rain and snowfall to fog and maybe even blinding sunlight or unstructured outside scenery. Each of these conditions reduce the pilots' ability to perceive visual cues in the outside world reducing his performance and finally increasing risk of mission failure and accidents, like for example Controlled Flight Into Terrain (CFIT). The basis for the presented solution is a fusion of processed and classified high resolution ladar data with database information having a potential to also include other sensor data like forward looking or $360^{\circ}$ radar data. This paper reports on a pilot assistance system aiming at giving back the essential visual cues to the pilot by means of displaying 3D conformal cues and symbols in a head-tracked Helmet Mounted Display (HMD) and a combination with synthetic view on a head-down MFD. Each flight phase and each flight envelope requires different symbology sets and different possibilities for the pilots to select specific support functions. Several functionalities have been implemented and tested in a simulator as well as in flight. The symbology ranges from obstacle warning symbology via terrain enhancements through grids or ridge lines to different waypoint symbols supporting navigation. While some adaptations can be automated it emerged as essential that symbology characteristics and completeness can be selected by the pilot to match the relevant flight envelope and outside visual conditions.
\end{abstract}

Keywords: DVE, ladar, pilot assistance, CFIT, landing aid, 3D conformal symbology, data fusion, situational awareness

\subsection{Operational Concept}

\section{INTRODUCTION}

Although brownout is the most prominent example of Degraded Visual Environment (DVE) it is by far not the only condition in which visual perception of the pilot is degraded by environmental factors. The most frequently encountered DVE condition is total darkness in areas with no or little man-made light sources, like Afghanistan. Depending on the operational area other DVE conditions, like fog, rain or snowfall, either separately or in combination with darkness are limiting the operational success of today's helicopter missions. It also has to be noted that the challenges of operating helicopters in DVE are not limited to landing. They extend over the complete range of flight phases from take-off, via taxing, hovering, cruise and nap-of-the-earth (NOE) flights to landing. These were also the tasks given to the NIAG report on helicopter operations at low altitude in $\mathrm{DVE}^{6}$. The challenges as well as the possible remedies may be quite diverse over the complete range of missions and DVE conditions. Brownout and whiteout stands out in this respect, as the usual approach is in good visual conditions (even when performed at night) which turn into a practically zero visibility condition in the last five to ten seconds of the landing phase. Performing missions in other DVE conditions have to be viewed differently as they typically do not have a good visibility phase but are operated in DVE conditions over the complete mission. One example stated here is a search and rescue mission performed at medium to heavy rain where degradation by rain is prevalent over the complete mission. Degradation hardly goes down to zero visibility but limited visibility and low contrast increase pilot's workload. Similarly a mission in an alpine environment even under visual flight rules (VFR) conditions can be quite challenging if visibility is at the lower limits (e.g. $800 \mathrm{~m}$ ).

The task of the system introduced here is to enlarge the safety margin and potentially also the possible application of visual flight to the boundaries of today's VFR regime. 


\section{SFERION - PILOT ASSISTANCE DURING THE ENTIRE MISSION}

SFERION is based on an open architecture and modular avionics. It utilizes an ARINC 653 SW framework and provides standardized and certifiable SW and HW building blocks. This cross-platform architecture concept allows configuring modular, scalable and tailored solutions according to platform configuration, operational requirement and budgetary constraints and upgrade already installed solutions by adding new functions and sensors.

Available solutions range from equipments to sub-system solutions. A variety of situational awareness functions are supported by different types of sensors combined and fused with information coming from database.

Figure 1 depicts the structure of the SFERION product family. Each of the included products such as SferiAdvise or SferiSense can be operated stand-alone or combined to an integrated sub-system.

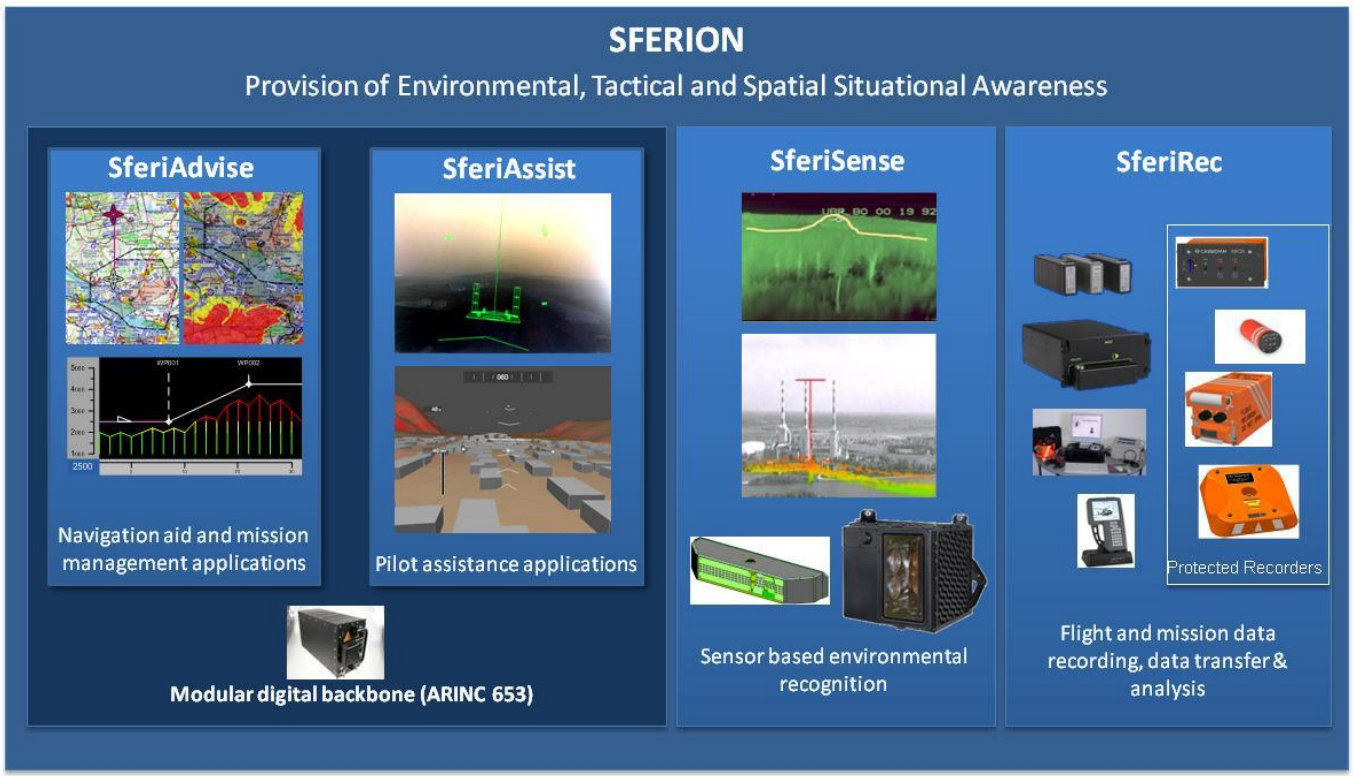

Figure 1. SFERION product families.

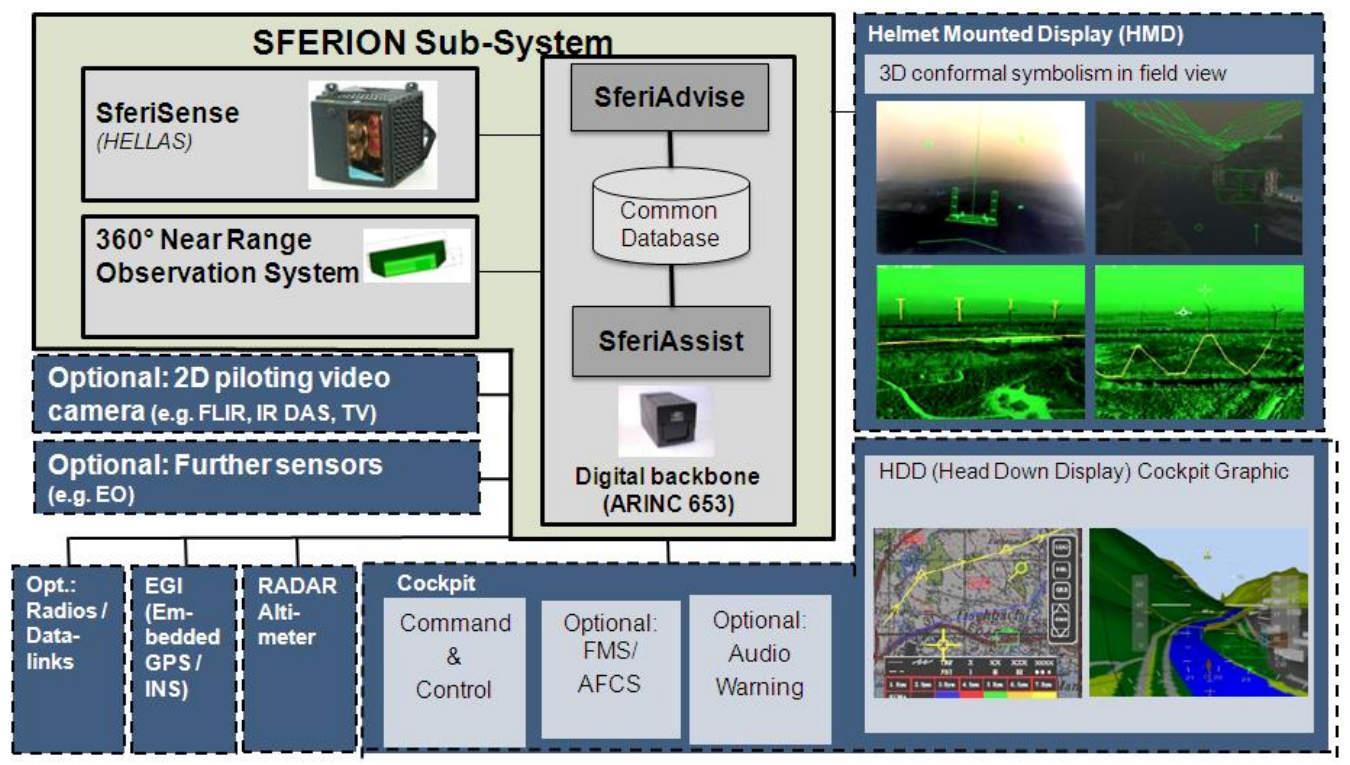

Figure 2. Typical SFERION sub-system architecture. 
SFERION combines SferiAdvise, SferiSense, SferiAssist and SferiRec to a high-end sub-system solution providing realtime information about the platform surrounding as well as tactical and spatial awareness, all enabled by fusion of sensor and database information.

The solution can be adapted to the Concept of Operations, the HMI philosophy and the crew concept of the specific platform of the operator. Due to the modular design of the system already existing and integrated equipments such as navigation system, radar altimeter or helmet mounted display can be directly re-used. Furthermore not all SFERION components need to be integrated at the same time. Hence, it is possible to integrate only a subset of the system, e.g. SferiSense (Obstacle Warning System) in the first step and later to extent the system in a second step, e.g. by SferiAssist.

An example of a typical SFERION sub-system configuration is depicted in Figure 2.

\section{3D SENSOR TECHNOLOGY / KEY PERFORMANCE IN DVE CONDITIONS}

The main sensor employed for the SFERION system is the SferiSense ladar, which is fully qualified as an obstacle warning system and in operational use on the German and Finnish NH90 helicopters. The sensor has a certified detection range of $725 \mathrm{~m}$ on a $5 \mathrm{~mm}$ diameter wire and has proven its capabilities over the complete range of DVE conditions from rain, via fog to snowfall and whiteout conditions. The sensor was designed to support low-level flight and hover and performs a classification of each 3D measurement point. The resulting classes displayed in the obstacle warning mode are wires, poles and isolated trees, all classified according to their geometrical properties in $3 \mathrm{D}$ space ${ }^{10}$. SferiSense operates at the eye-safe wavelength of $1.54 \mu \mathrm{m}$ and scans its field-of-view up to a distance of $1200 \mathrm{~m}$ with $3 \mathrm{~Hz}$.

Theoretical analysis as well as flight tests showed that SferiSense can detect wires at any angle of incidence, at the extreme even when flying parallel to the wire. Flight tests also showed that performance degradation due to rain is almost negligible for light and medium rain. Even for heavy rain with a rate of $10 \mathrm{~mm} / \mathrm{h}$ detection range on a $5 \mathrm{~mm}$ wire is still exceeding $500 \mathrm{~m}$. Special processing/filtering techniques for snowfall were designed and implemented and verified during qualification flights in medium snowfall. The binocular optical design ${ }^{10}$ together with an active receiver management also minimizes artifacts due to fog down to visibilities of $800 \mathrm{~m}$.

The system was also flight tested hovering in whiteout and showed its continued performance detecting terrain and obstacles in the close range even through the whiteout cloud. Ground and flight tests in brownout were performed with the goal to push the sensor performance to the physical limits. In the literature ${ }^{4,5}$ differing values for dust concentrations created by helicopters in brownout can be found. The most commonly used definitions relate brownout condition with dust concentration as e.g.

- light

- medium

$0.5 \mathrm{~g} / \mathrm{m}^{3}$

- heavy

$2.0 \mathrm{~g} / \mathrm{m}^{3}$

$3.0-3.5 \mathrm{~g} / \mathrm{m}^{3}$

Although widely used this purely concentration based definition neglects the fact that particle size distribution is a second factor in the equation evaluating extinction coefficients under the respective conditions. The smaller the dust particles are, the higher the extinction coefficient will be. For very fine dust extinction coefficients can go up as far as shown in Table 1. The above stated values assume a perfect lidar/ladar system without losses in the optical path or due to overlapping. Nevertheless this theoretical data is in line with own measurements and consistent with published data from other sensors ${ }^{5}$. The jump from light to moderate brownout conditions according to the above definition is quite extreme. For a comparison, the extinction coefficient inside a cumulus cloud ${ }^{8}$ is typically between 200 and $300 \mathrm{~km}^{-1}$. The calculated two-way losses shown in Table 1 clearly show that for so called moderate or heavy brownout conditions, detection even of an extended obstacle in an operationally relevant distance (beyond $20 \mathrm{~m}$ to $30 \mathrm{~m}$ ), is not feasible.

To understand why lidar systems, including SferiSense, do show a dust look-thru capability a closer look at the nature of brownout clouds is necessary. The aerosol concentration is much more inhomogeneous than for example in heavy fog. Local dust concentrations inside this extremely turbulent cloud may vary by an order of magnitude. This on the other hand results in patches or windows with a much higher visibility than the general, potentially averaged dust concentration may suggest. Therefore an optical sensor operating in the $1.5 \mu \mathrm{m}$ region will have phases of total blocking by brownout but there is a good chance to find a window when data acquisition is possible again. 
Table 1. Brownout conditions, related extinction coefficients and derived two-way attenuation of a signal.

\begin{tabular}{|l|l|l|l|l|}
\hline Brownout condition & $\begin{array}{l}\text { dust } \\
\text { concentration } \\
{\left[\mathbf{g} / \mathbf{m}^{3}\right]}\end{array}$ & $\begin{array}{l}\text { maximum } \\
\text { extinction } \\
\text { coefficient }[\mathbf{1} / \mathbf{k m}] \\
\text { @ } \mathbf{1 . 5} \boldsymbol{\mu m} \mathbf{f o r} \\
\text { very fine dust }\end{array}$ & $\begin{array}{l}\text { resulting 2-way } \\
\text { transmission } \\
\text { attenuation across } \\
\mathbf{2 0} \mathbf{~ m} \text { of dust }\end{array}$ & $\begin{array}{l}\text { resulting 2-way } \\
\text { transmission } \\
\text { attenuation across } \\
\mathbf{3 0} \mathbf{~ m} \text { of dust }\end{array}$ \\
\hline light & 0.5 & 150 & $2 * 10^{-2}$ & $10^{-4}$ \\
\hline moderate & 2 & 600 & $4 * 10^{-8}$ & $2 * 10^{-16}$ \\
\hline heavy & 3 & 900 & $2 * 10^{-12}$ & $4 * 10^{-24}$ \\
\hline
\end{tabular}

\section{PROCESSING}

SFERION comprises a common digital backbone for hosting the SferiAssist and symbol generator functionality and for providing the required interfaces towards the sensors, the avionics environment and the displays. The AIRBUS Defence and Space digital backbone is a modular avionics processing unit which is configured according to the processing and interface requirements derived from the specific SFERION configuration (e.g. processing capacity, memory capacity, graphic processing capacity) and avionics system requirements (e.g. interfaces). The digital backbone provides all benefits of an Integrated Modular Avionics (IMA) platform designed for operation in a certified airborne environment. It offers tailoring to its intended use and thus optimization of cost. Its ruggedized hardware is qualified in accordance to RTCA DO-160 and MIL-STD-810. It complies with the challenging EMC requirements of modern aircraft that are using composite materials.

The digital backbone is future-proof due to its high performance processing capabilities, ARINC 653 real-time operating systems (RTOS, incl. Board Support Package BSP), extensive BIT support and data loading capabilities (ARINC 615A). Furthermore it can be easily converted applying additional plug-in modules for avionics data bus integration (ARINC 664, ARINC 429, MIL-STD-1553B) or graphics applications.

Based on the existing AIRBUS Defence and Space HW module catalogue, the detailed interface requirements of aircraft and the growth potential to be considered, the detailed HW architecture can be flexibly defined.

Processing of data accumulation and fusion is performed in a so called Quadtree structure hosted on the digital backbone. This Quadtree allows for efficient adaptation of the resolution i.e. the Level of Detail (LOD) for display and accounts at the same time for the range dependent accuracy of the 3D-Sensor data. Figure 3 shows the structuring of different LODs. Currently SFERION uses LODs from 0 through 5, resulting in post spacing between $128 \mathrm{~m}$ and $4 \mathrm{~m}$. Details of 3D data classification and LOD processing can be found in ${ }^{3}$.
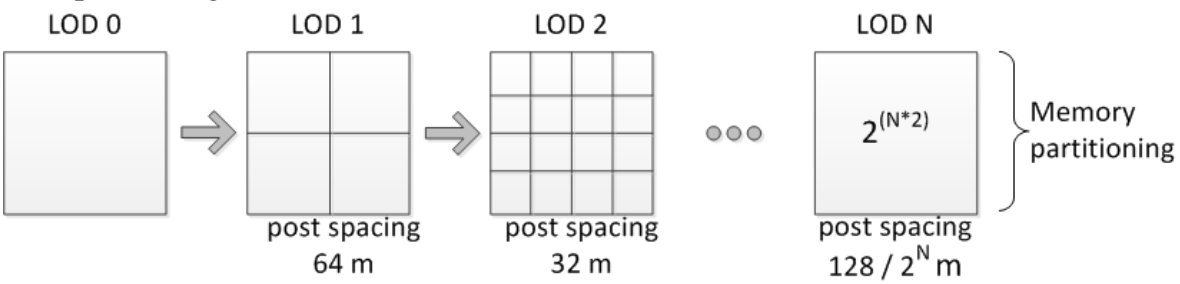

Figure 3. Quadtree memory structure employed in SFERION processing.

Although information typically originates from different sources it is necessary to display only one set of information to the pilot ${ }^{6}$. In SFERION processing fusion of 3D information takes place in different layers or silos. The first step is the fusion of classified objects from different sensors and databases. It is done by combining matching objects at the same or similar position. The challenges for this step but also for all following steps are different accuracies of measured object positions and features coming from different sensors (incl. navigation unit accuracy) and databases ${ }^{6}$. For the fusion of classified 3D objects (WIREs, POLES, isolated TREEs and man-made objects ${ }^{3}$ ) a position and property matching technique is employed. Although this technique is successful in combining objects most of the time, in case of doubt a doubling of the objects has to be accepted. The right image in Figure 7 shows an example in which the power line crossing the valley is detected and classified by the sensor with high resolution and also given in the obstacle database with lower resolution. Although the two hypotheses match well in their property direction, the difference in height does not allow for a single fused object. Therefore in this case two obstacle symbols have to be displayed to ensure flight safety. 
The next layer is the 2.5 or 3D terrain fusion, i.e. the fusion of classified measured terrain data points with terrain DB data. Fusion in the Quadtree structure takes care of the issues of different resolutions, specific margins of errors in data sources as well as known weaknesses of specific data sources. The flight proven fusion scheme for 2.5 or 3D terrain fusion employed in the SFERION system is to use sensor data enhancing database data in the mid to near range (below $1000 \mathrm{~m}$ or less). Thereby terrain database is used in sensor void areas or in out of FOV areas of sensor data. The left hand image of Figure 11 shows the areas where sensor data is used (light and dark green terrain in the middle) versus the areas where database data is used (olive green terrain on left hand side in front of power line).

The subsequent layer is the fusion of unclassified 3D data points originating from the sensor(s) with objects or structures in the database. For reasons of effectiveness in real time processing, data fusion at this point is also performed in the Quadtree structure, not as a point by point matching.

The resulting fused information serves as basis for all visualization processes.

\section{HUMAN MACHINE INTERFACE - HMI}

The main goal of the HMI is to reduce pilots' workload in all phases of flight. For doing this a combination of 3D conformal cues and symbols displayed on a head-tracked HMD with a synthetic view displayed on the head-down MFD is used. Extensive HMI workshops and HMI loops in a fixed based simulator as well as flight tests have shown that the preferred usage of either display unit depends on the phase of flight, the current environmental conditions and the height above ground. The outcome of these tests shows that during cruise flight in a safe altitude above ground synthetic view on MFD is used for navigation support and increased situational awareness. The closer the mission drives the pilot to the obstacle scenery and the terrain the more a head-up eyes-out support with 3D conformal symbology on the head-tracked HMD is preferred. This is also consistent with the findings in other publications ${ }^{6}$. The HMI workshops also resulted in the definition of several decluttering and mode adaptation possibilities which were indicated as necessary by pilots when using the SFERION system in different DVE conditions. Figure 4 gives an impression how different perception of the environment can be, even at the same time of day and flying at the same altitude.
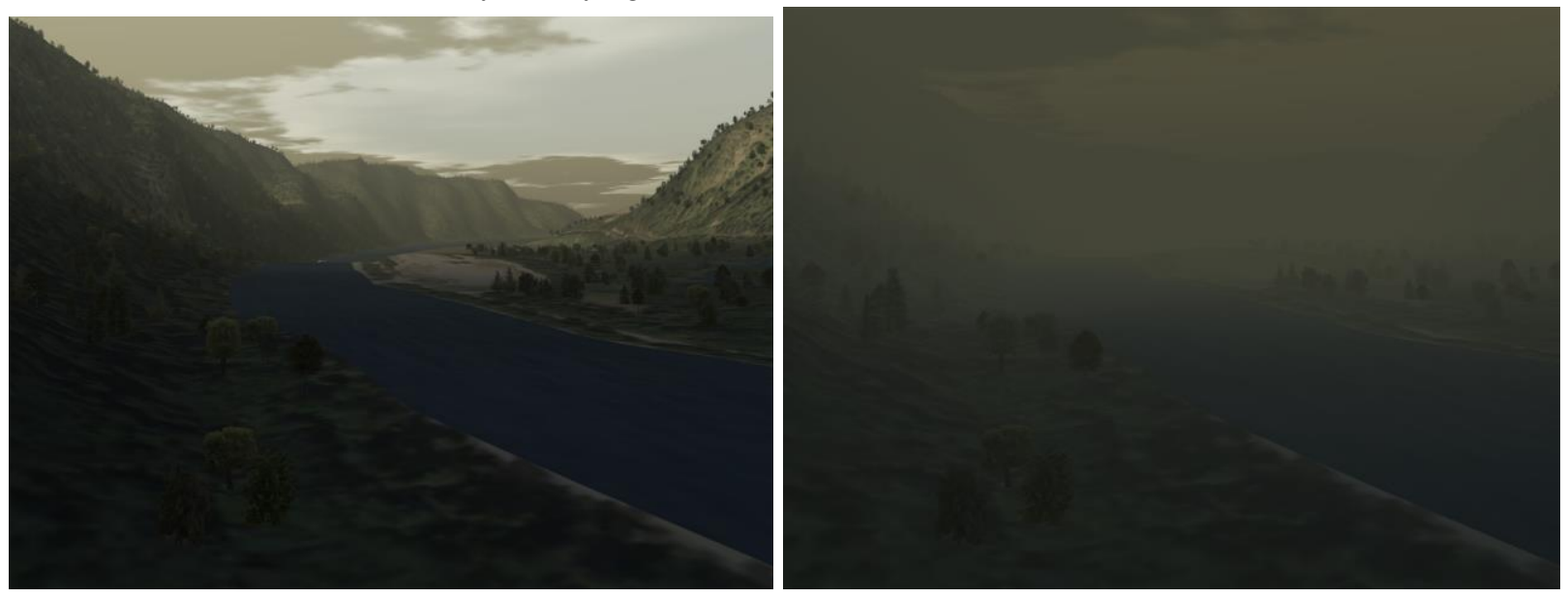

Figure 4. Simulated cockpit view of identical point with varying visibility conditions (12 km on the left, $1200 \mathrm{~m}$ on the right), seen at a height above ground of $80 \mathrm{ft}$.

While both conditions are clearly within VFR limits, risk and potential need for augmentation of pilots' visual perception are extremely different. With a good visibility the factor requiring pilots' attention is mainly the potential decrease of contrast due to the time of day (in this example dawn). Flying a mission under these conditions may only be challenging because looking out for obstacles in unknown terrain can increase workload. If the same mission has to be conducted under visibility conditions around $1200 \mathrm{~m}$ most of the visual cues are lost. Only in the close range in front of the helicopter some cues on the ground are left. Picking up obstacles like wires under these conditions is almost impossible. If mission constraints or simply a required clearance to the cloud ceiling prevents flying at higher altitudes, pilot support functionalities are needed. 


\subsection{Information Displayed on HMD}

Information displayed on the HMD is based on fused data from the high resolution 3D ladar sensor SferiSense and database information. The goal of HMD display is to augment pilots' visual perception. The SFERION system is not intended to present a fully synthetic imagery on the HMD. This would mask the remaining outside view the pilot still has with all the consequences related to this, especially concerning certification. On the other hand pilots' workload becomes quite extreme when missions have to be conducted in low-level flight, in a challenging environment under DVE conditions.

SFERION offers a set of display possibilities on the HMD to support pilots' visual perception. Situational awareness and clearance from the ground (preventing CFIT) can be increased by several functionalities. The first one is already in operational use on the German and Finnish NH90 helicopters employing the so called safety-line ${ }^{10}$. This safety-line depicts the calculation of different evasion profiles within the ladar field-of-view in a simple line structure. Pilot's rule is to simply keep the flight vector above the safety-line to be safe. Figure 5 shows a snapshot of the safety-line taken at a low-level flight crossing a wind farm.

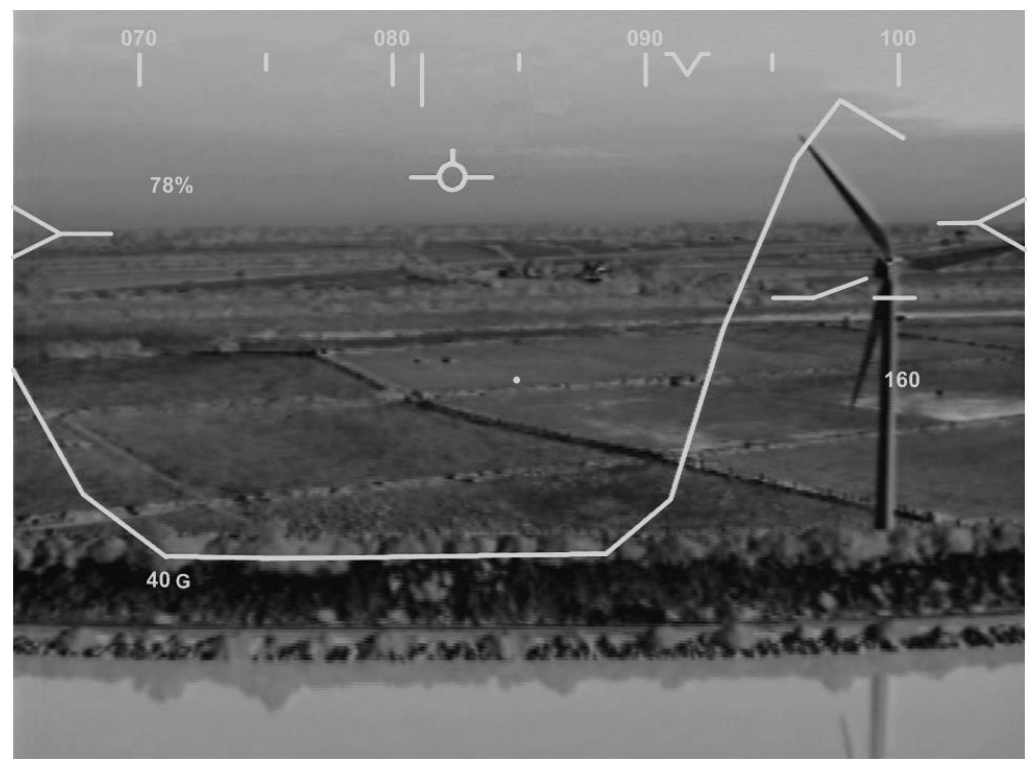

Figure 5. Safety-line used for preventing CFIT and object strike overlaid on a FLIR image while crossing a wind farm.

While the safety-line supports pilotage by displaying possible evasion paths it does not give a direct depiction of the terrain in front of the helicopter. It may be desired to directly augment the perception of terrain and landscape shape though, even beyond the sensor range. This is supported by SFERION by optionally displaying a terrain grid combined with or independent from contour lines. The terrain grid is a depiction of the fused terrain information coming from terrain databases, like DTED or HRTI, and classified ladar sensor terrain data. The information is fused on different LODs as described in chapter 4. Figure 6 shows an example of the grid representation. Because the grid information tends to be quite dominant it is typically displayed darker than the other symbology on the HUD. Obstacle symbology on the other hand has to be displayed at maximum brightness. The right side of Figure 6 shows the same HMD view with the addition of contour lines. Such contour lines are also called ridge lines in other publications ${ }^{9}$. The innovation is that the contour lines are based on the fused information and therefore can also be applied for structures in closer ranges with higher resolution thanks to the active sensor data. 

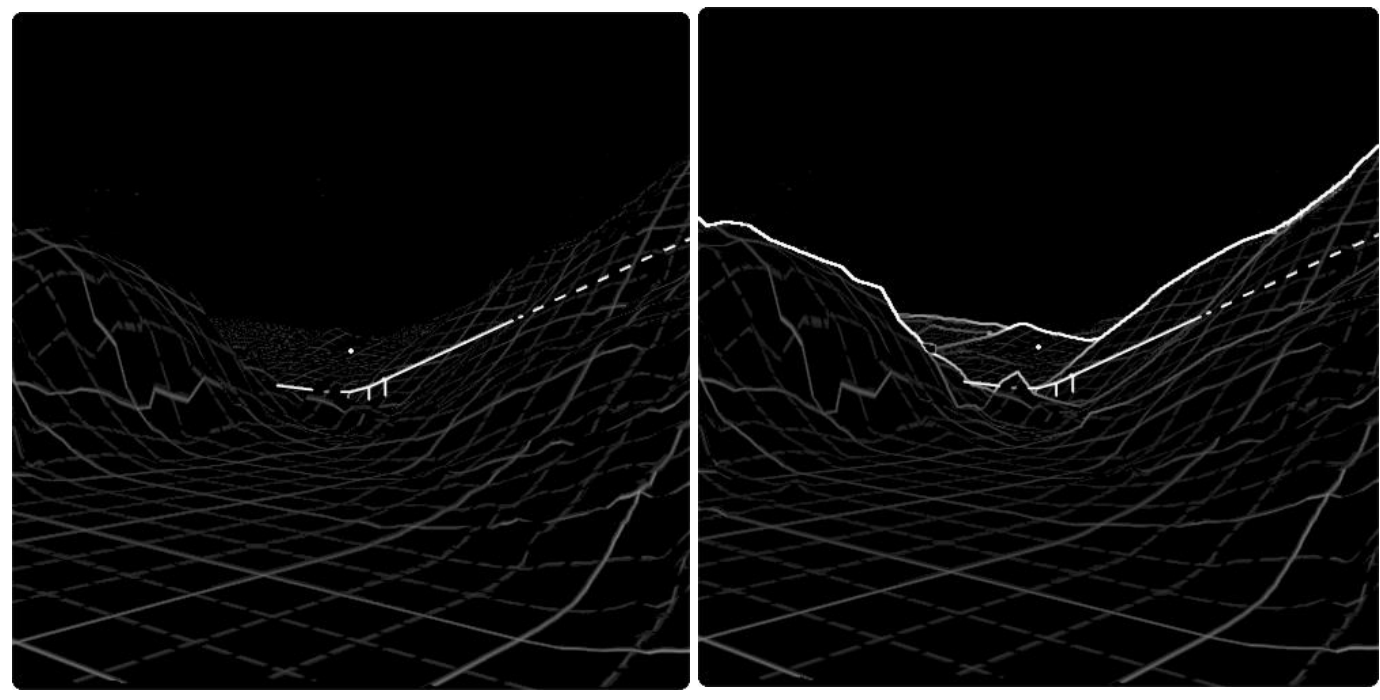

Figure 6. Display of terrain grid (left image) and terrain grid with contour lines (right image).

An outcome of the HMI trials and workshops was an additional decluttering possibility for the terrain grid and the contour lines in the foreground. Displaying a conventional grid in DVE flights with limited visibility pilots' view on remaining terrain features and textures was cluttered by this grid structure. On the other hand the grid was seen as very helpful in distances where the visual perception was obstructed. This led to the concept of "culling", i.e. the pilots' possibility to shift the beginning of grid and contour line display further away from the helicopter position. Figure 7 shows an example where the beginning of terrain grid display was shifted away by $800 \mathrm{~m}$.
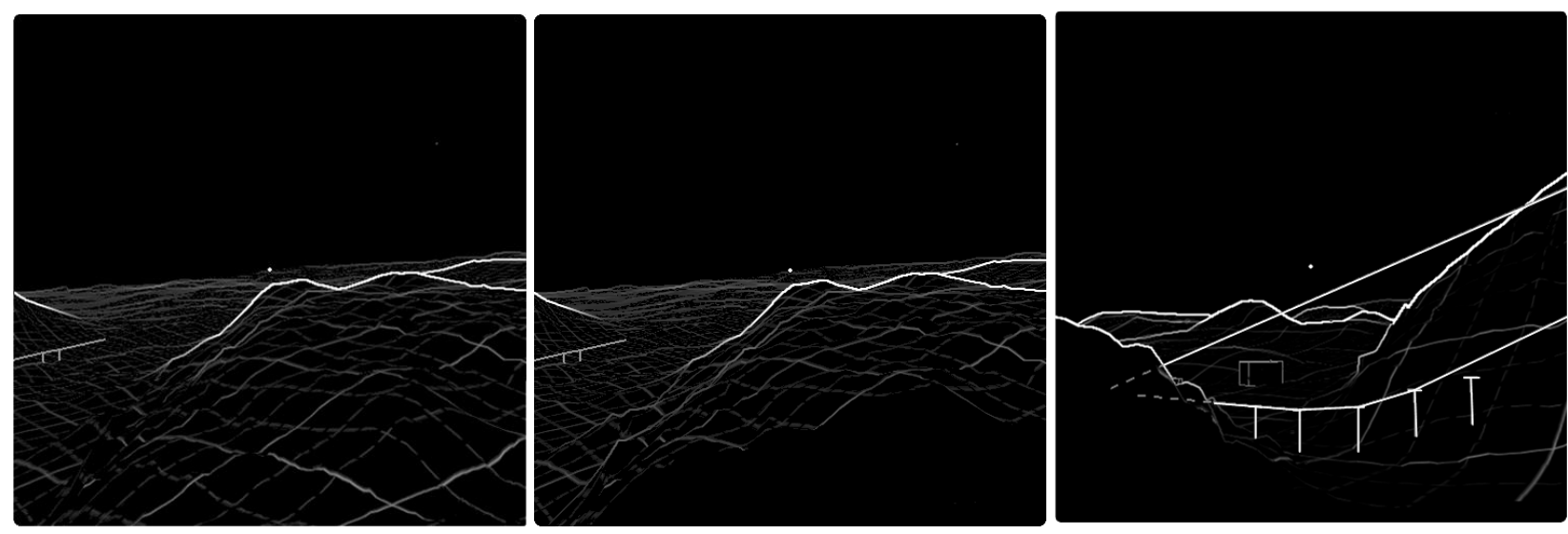

Figure 7. Display of complete terrain grid with contour lines (left image) and terrain grid with contour lines with a shifted start of display (right images).

Figure 8 shows the advantages of a terrain augmentation by a grid and contour lines with a shifted start of display of this conformal symbology (culling). 


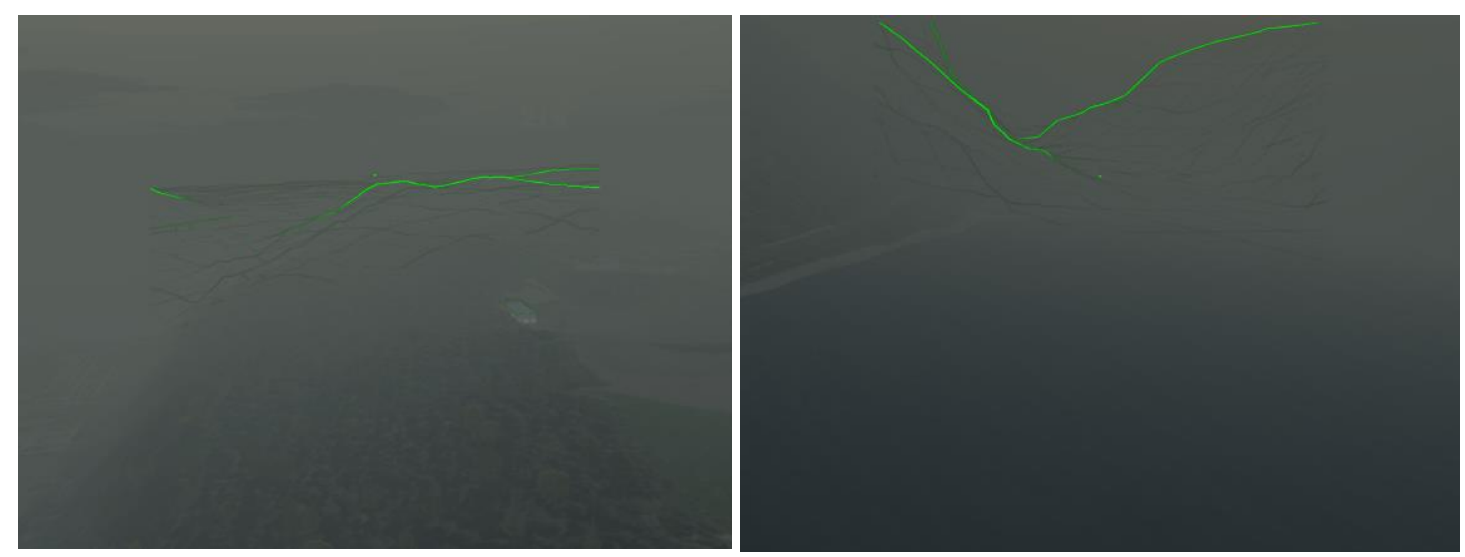

Figure 8. Display of terrain grid with contour lines with a shifted start of display overlaid on outside view at a visibility of $1200 \mathrm{~m}$.

As already shown in Figure 6 the standard SferiSense obstacle symbology ${ }^{10}$ (straight line for wires and "T" symbol for poles) is always included in the symbology set.

Although the previous figures only show so called 3D conformal symbology without a standard 2D symbology this is not the operational case. 3D conformal symbology is intended to augment pilots' view by symbology, trying to give back some cueing that was taken from them by DVE. For platforms that already have a 2D symbology generated by their HMD this can be used with minor modifications. In these cases careful attention has to be taken to match 2D and 3D information. For platforms not having an HMD so far, 2D symbology can also be generated by SFERION. Figure 9 shows an example for the scenery displayed on the left hand side of Figure 8.

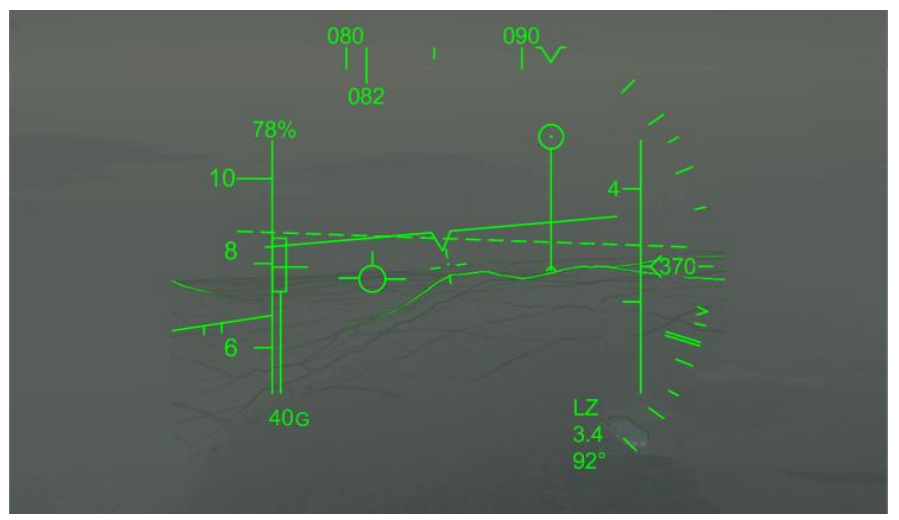

Figure 9. 3D conformal symbology (terrain grid with contour lines) overlaid with 2D symbology supplemented with a display of the waypoint denoting the landing zone.

Navigation is supported by 3D conformal display of waypoint symbols placed on the fused terrain. The shape of these symbols is consistent with the waypoint symbol shape defined for head-down route planning and digital map systems. Waypoints can be displayed either as fly-by waypoint with a height that remains constant in the field of view or as flyover waypoints with a height depicting the pre-planned fly-over height. Figure 9 shows an example of a landing zone waypoint symbol combined with terrain grid, contour lines and 2D symbology.

For final approach and landing in DVE a special landing symbology is displayed. This symbology consists of specific guidance markers leading the pilot towards the landing zone as well as a sensor based high-resolution grid structure in the landing zone. On this grid synthetic cues are placed. The details of this symbology including remote slope detection and display were described in more detail before ${ }^{2}$. Figure 10 shows an example seen from a distance of approx. $70 \mathrm{~m}$ from the selected touch-down point. 


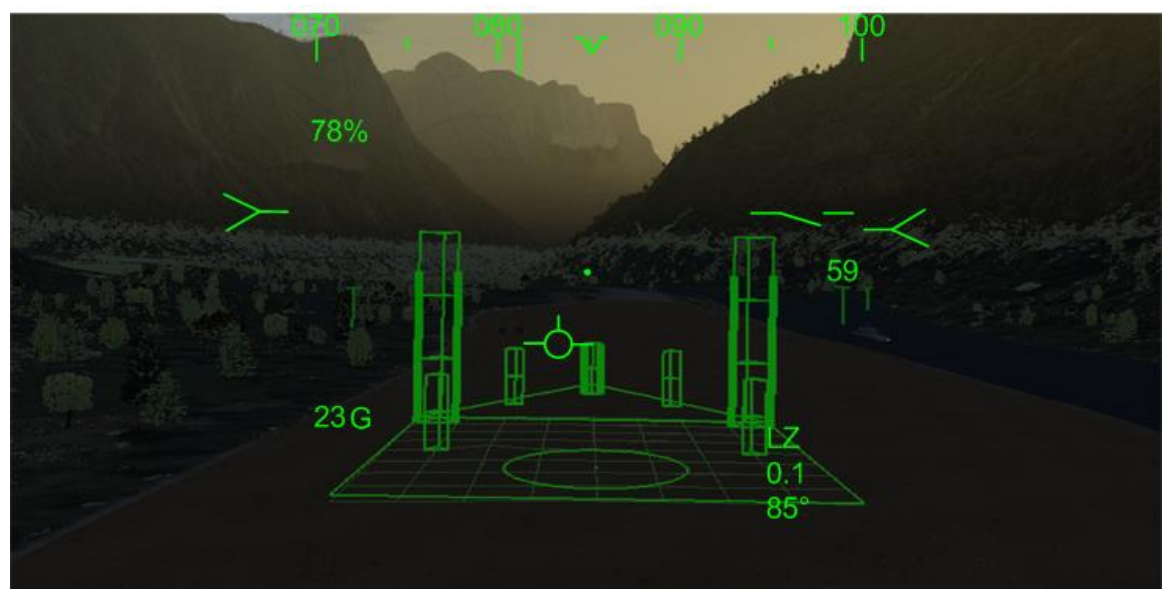

Figure 10. 3D conformal landing symbology with doghouse, high resolution grid and synthetic cues as seen from approx. 70 $\mathrm{m}$ distance just before entering the brownout cloud.

\subsection{Information Displayed on HDD}

The synthetic vision system on the Head-Down Display (HDD) was preferably used to navigate and increase situational awareness when flying above but potentially close to the obstacle scenery. The main advantages of synthetic vision are its ability to display high resolution imagery in color without the need to ensure sufficient transparency of the display. The synthetic vision may be displayed either on a separate display screen or overlaid by helicopter PFD information.

Although display of a photorealistic textures, e.g. satellite imagery as texture, is possible, usage of obviously synthetic textures is done by intend. Pilots must be able to distinguish synthetic parts from real world or camera views. This paradigm is in line with requirements given by some national certification authorities.

Data source for the synthetic vision is the same as for the HMD 3D conformal symbology - the classified 3D sensor information fused with available database information. Details of the classification process as well as the display generation process were already presented earlier ${ }^{3}$. In addition to the symbology displayed on the HMD display of so called manmade objects, e.g. houses, supports navigation and situational awareness. Similarly the display of database information on streets and rivers or creeks enables landmark navigation in a variety of missions. Figure 11 shows on the left hand side an example of a synthetic vision profile differentiating different terrain features (agricultural areas vs. forest, etc.). Streets are visualized in grey, database-sensor fused obstacles are displayed in amber.
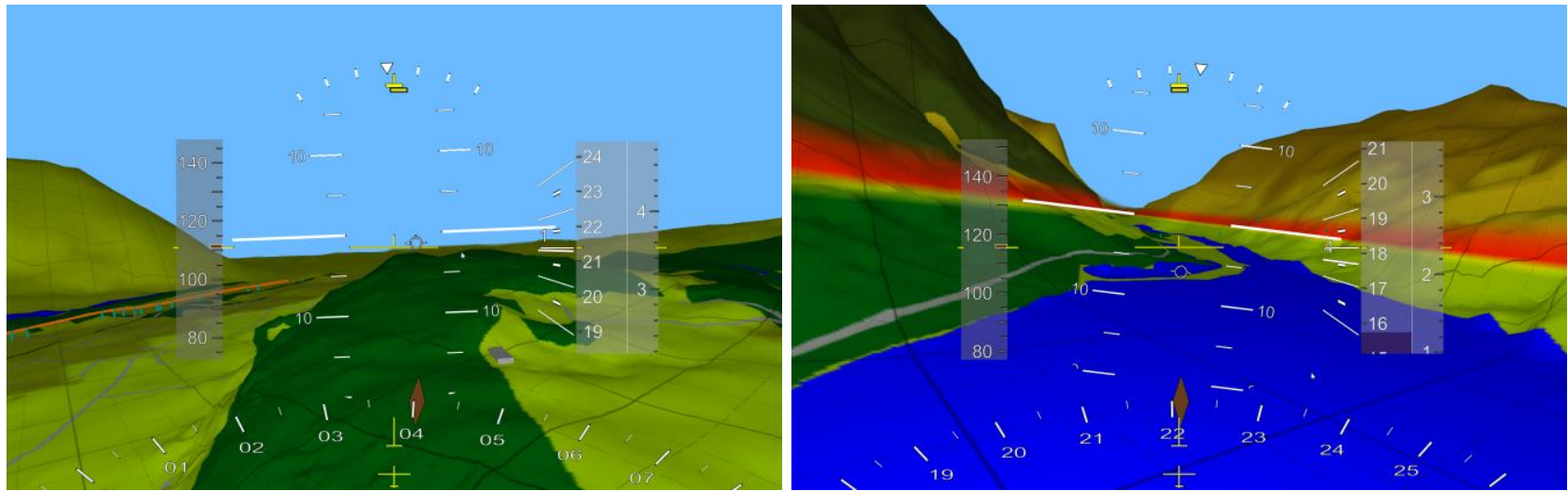

Figure 11. 3D sensor enhanced synthetic vision display on HDD.

Depending on the flight profile and the environment different color schemes can be employed. Figure 11 shows on the right hand side an example of a synthetic vision profile with color coded height information. To avoid cluttering with too many red areas the standard HTAWS color coding cannot be applied. In this example only the height band at the helicopter height or slightly above is coded in red to avoid supersaturation. 
The grid displayed on the terrain has a fixed but configurable spacing providing additional distance cues. Figure 11 shows that classified fused obstacles are also displayed in the synthetic view head-down. The same is true for waypoints, manmade objects and tactical information.

Feature selection and decluttering modes can be selected independently for HMD and HDD display. This allows e.g. deselection of specific features, like man-made object display on the HMD to avoid cluttering, while having this information displayed on the HDD for better situational awareness.

Current work in this field goes in the direction of further tailoring displayed information to the pilots' needs in the specific situation as well as combining synthetic vision display with direct imaging systems like a distributed aperture system (DAS). While the DAS imagers typically show a high resolution photorealistic image, they typically lack specific information like the 3D content or obstacle information and they are subject to degradation by environmental conditions. Both of which could be complemented by adding synthetic parts in the 2D DAS images.

\section{SUMMARY}

Within its product family SFERION Airbus Defence and Space has developed a system concept for a sensor based DVE pilot assistance system. It demonstrates the benefits of fusing 3D sensor information coming from different sources with database information. The process of generating a single concise picture to the pilot is based on the fusion of classified information hosted in a digital backbone core processor. This core processor finally generates low latency display information for the HMD as well as for the HDD. While the synthetic vision display on the HDD was preferably used for navigation support and gaining situational awareness while flying above the obstacle scenery, HMD 3D conformal symbology was the preferred choice for low level operations in DVE, including landing. Coping with the multitude of different DVE conditions a set of features for tailoring 3D conformal symbology to the actual needs in the current condition and mission profile was developed and successfully tested.

\section{ACKNOWLEDGEMENTS}

The authors would like thank the German BAAINBw for their support and investment during the demonstrator study "Sensor based Landing Aid II" (SeLA-II) regarding HMI workshops as well as HMI and flight trials on the UH-1D test platform MAT.

\section{REFERENCES}

[1] Waanders, T., Münsterer, T. and Kress, M., "Sensor Supported Pilot Assistance for Helicopter Flight in DVE", Proc. SPIE 8737 (2013).

[2] Münsterer, T. Kress, M. and Klasen, S., "Sensor based 3D conformal cueing for safe and reliable HC operation specifically for landing in DVE", Proc. SPIE 8737 (2013).

[3] Schafhitzel, T., Hoyer, M. and Völschow, P., "Increasing Situational Awareness in DVE with Advanced Synthetic Vision", Proc. SPIE 8737 (2013).

[4] Cross, J., Schneider J. and Cariani P., "MMW Radar Enhanced Vision Systems: The Helicopter Autonomous Landing System (HALS) and Radar Enhanced Vision System (REVS) are rotary and fixed wing enhanced flight vision systems that enable safe flight operations in degraded visual environments", Proc. SPIE 8737 (2013).

[5] Trickey, E., Church, P. and Cao X., "Characterization of the OPAL Obscurant Penetrating LiDAR in various Degraded Visual Environments”, Proc. SPIE 8737 (2013).

[6] NATO Industrial Advisory Group (NIAG), "Final Report of NIAG SG167 on Helicopter Operations at Low Altitude Degraded Visual Environment (DVE)", 25 July 2013, NIAG-D(2013)0014, AC/225(VL)D(2013)0001.

[7] Wolfe, W.L. and Zissis G.J., ed., [The Infrared Handbook], The Infrared Information and Analysis (IRIA) Center, Environmental Research Institute of Michigan, 1978.

[8] Stratton Park Engineering Company, "Extinctiometer Theory", 2012, < http://www.specinc.com/extinctiometertheory> (30 March 2014).

[9] Lemoine, O., François, J.-F. and Point, P., „Contribution of TopOwl Head Mounted Display System in Degraded Visual Environments", Proc. SPIE 8737 (2013).

[10] Seidel, C., Samuelis, C., Wegner, M., Münsterer, T., Rumpf, T. and Schwartz, I., "Novel approaches to helicopter obstacle warning", Proc. SPIE 6214, 621406 (May 19, 2006). 